Оригинальная статья/Original article

УДК 33.338.2

DOI: http://doi.org/10.20914/2310-1202-2016-2-334-338

Моделирование финансовых результатов на основе факторного анализа

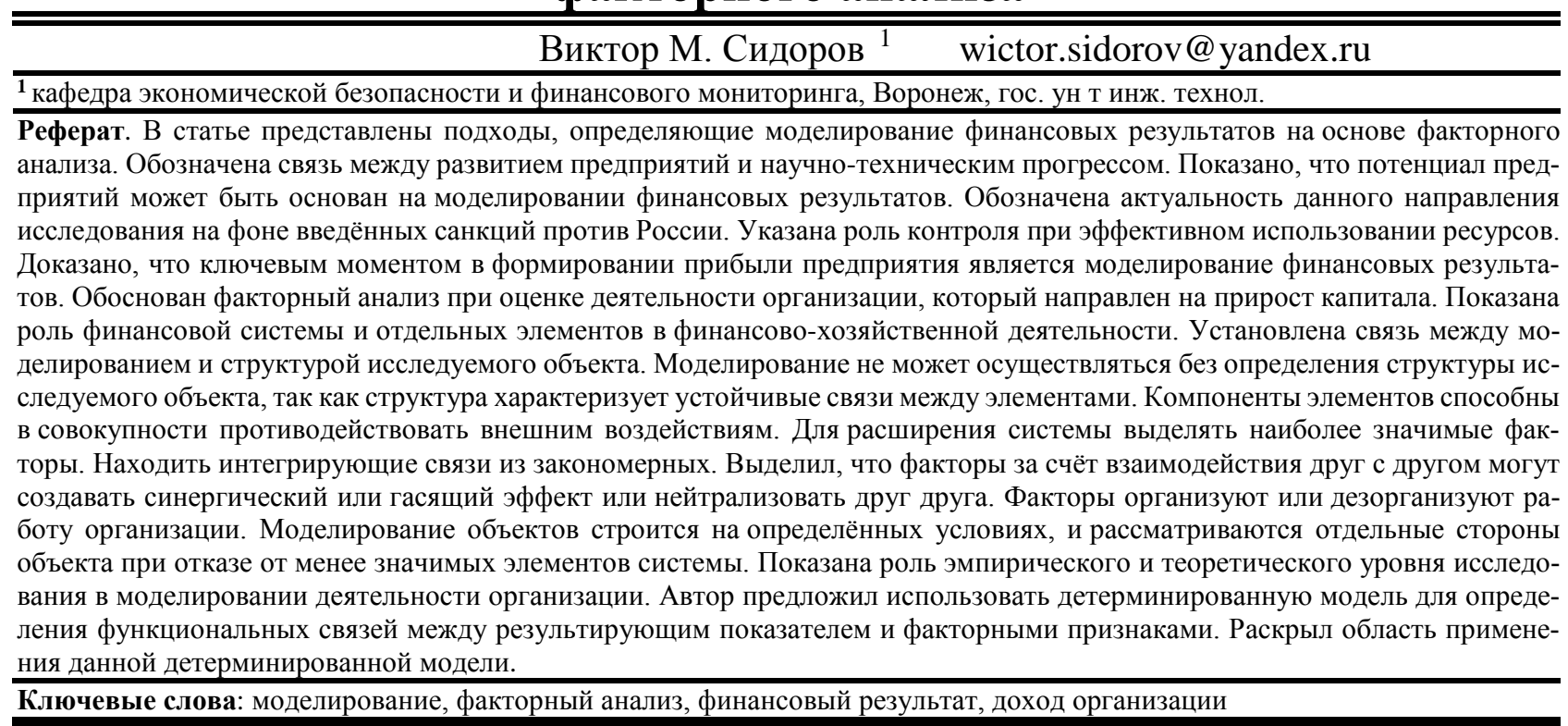

\title{
Design of financial results on the basis of factor analysis
}

\begin{abstract}
Victor M Sidorov $^{1} \quad$ wictor.sidorov@yandex.ru
$\overline{1}$ Department of economic security and financial monitoring, Voronezh state university of engineering technology

Summary. Approaches are presented in the article, qualificatory the design of financial results on the basis of factor analysis. Connection marks between development of enterprises and scientific and technical progress. It is shown that potential of enterprises can be based on the design of financial results. Actuality of this direction of research marks on a background the entered approvals against Russia. A control role is indicated at the effective use of resources. It is well-proven that a key moment in forming of profit of enterprise is a design of financial results. A factor analysis is reasonable at the estimation of activity of organization, which exposed an application of this determined model domain. is sent to capital augmentation. The role of the financial system and separate elements is shown in to financially-economic activity. Connection is set between a design and structure of the investigated object. A design cannot come true without determination of structure of the investigated object, because a structure characterizes steady connections between elements. The components of elements are able in totality to counteract to external influences. For expansion of the system to distinguish the most meaningful factors. To distinguish integrating connections from appropriate. Distinguished, that factors due to co-operating with each other can create a synergistically or extinguishing effect or neutralize each other. Factors will organize or will disorganize work of organization. The design of objects is built on the certain terms, and separate parties of object are examined at abandonment from the less meaningful elements of the system. The role of empiric and theoretical level of research in the design of activity of organization is shown. An author suggested to use the determined model for determination of functional connections between a resulting index and factor signs. Exposed an application of this determined model domain.
\end{abstract}

Keywords: factor analysis, financial result, acuests of organization

\section{Введение}

Развитие экономики предприятий зависит от научно-технического прогресса, уровня подготовки инженерно-технических кадров, поступательного роста отраслей промышленности и в целом связано с техникообразовательным процессом в обществе [1].

Вместе с тем, реализация потенциала коммерческих предприятий невозможна без

Для цитирования

Сидоров В. М. Моделирование финансовых результатов на основе факторного анализа // Вестник ВГУИТ. 2016. № 2. С. 334-338. doi:10.20914/2310-1202-2016-2-334-338

334 формирования основных подходов в системе моделирования финансовых результатов на основе факторного анализа. Это связано с влиянием как внешних, так и внутренних факторов. Причём в современном обществе все большее влияние оказывают внешние факторы, связанные с обострением противоречий между ведущими государствами мира в борьбе за сферы влияния, экономические ресурсы, сегменты рынка.

For citation

Sidorov V. M. Design of financial results on the basis of factor analysis. Vestnik VSUET [Proceedings of VSUET]. 2016. no. 2 pp.334-338. (in Russ.). doi:10.20914/2310-1202-2016-2-334-338 
Введённые санкции против РФ заставляют предприятия функционировать в сложных экономических условиях, когда возникают трудности при обеспечении поставок комплектующих изделий, в получении кредитов, необходимых для модернизации и реконструкции производственных мощностей.

В этих условиях, как никогда, возрастает роль контроля за основными составляющими производства и реализации продукции [2]. Контроль необходим для определения, насколько эффективно используются основные, оборотные средства, трудовые ресурсы.

\section{1 Моделирование финансовых результатов}

Моделирование финансовых результатов на основе факторного анализа становится ключевым фактом в оценке доли влияния вышеперечисленных показателей.

Коммерческий финансовый результат выражается через прибыль, убыток и точку безубыточности [3]. Также он является комплексным показателем, характеризующим эффективное использование имеющих в распоряжении предприятия ресурсов. Для ведения успешной финансово-хозяйственной деятельности необходимо, чтобы полученный финансовый результат превышал произведённые расходы, а это, в свою очередь, свидетельствует об эффективности производства [4].

Финансовый результат непосредственно связан с бухгалтерским учётом, который отражает экономическую деятельность хозяйствующего субъекта. Это позволяет формировать динамику результирующих экономических показателей.

Планирование финансово-хозяйственной деятельности является основным моментом в работе предприятий [5]. Данное планирование основывается на факторном анализе.

С помощью факторного анализа находят, за счёт каких фактов происходит формирование прибыли коммерческой организации.

Определяют наиболее значимые факторы, оказывающие существенное влияние на формирование прибыли организации.

Выделение факторов наибольшего влияния на конечные результаты является наиболее важным моментов в деятельности предприятия. От этого во многом зависит успешность финансово-хозяйственной деятельности и перспективы развития в целом.

В этих условиях большую роль играет прирост капитала предприятия, который формируется также и за счёт нераспределённой прибыли. Все в целом приводит к потребности моделирования финансовых результатов. Для этого необходимо обеспечивать переход от исходной факторной к конечной факторной системе. Причём факторная система строится на основе представления результирующего показателя в виде алгебраического выражения.

Вместе с тем, необходимо учесть, что моделирование строится не только на фиксации результатов, но и на обновлении [6]. Причём обновление инвариантных финансовых результатов строится на инновационных связях [7].

В понимании «система» решающая роль принадлежит значению слова элемент. Без него сама система теряет эвристический смысл. При данном рассмотрении элемент есть неразложимый компонент системы. В нашем случае факторы выступают в качестве элементов системы.

\subsection{1 Выбор структуры моделирования}

Моделирование не может осуществляться без определения структуры исследуемого объекта, так как структура определяет устойчивые связи между элементами. Причём сам характер связи внутри системы определяют элементы. Отсюда необходимо знание об особенностях функционирования конкретного объекта исследования, экономических процессах, происходящих при выполнении отдельных производственных операций.

Надо учитывать, что по своей значимости для системы связи элементов могут быть существенными или малозначимыми. Закономерные связи элементов определяют структуру предприятия. Между их компонентами присутствуют связи, которые способны противодействовать в течение определённого времени негативным воздействиям как внешней, так и внутренней среде. Близость таких элементов способствует исключению отдельных элементов или, наоборот, создаёт условия для включения отдельных компонентов в систему.

Все вышеперечисленное позволяет вносить или исключать, с точки зрения эксперта, отдельные составляющие в процессе моделирования экономических объектов. Это способствует выделению значимых факторов, учитывающих специфику деятельности предприятия.

При моделировании необходимо из закономерных связей выделять интегрирующие, которые, с точки зрения экономического подхода, характеризуют процесс производства и реализации товаров, работ, услуг.

\subsection{2 Роль факторов при моделировании}

В задачу экономического исследования входит расширение самой финансовой системы 
за счёт вовлечения в неё значимых факторов и определения доли их влияния на результирующий фактор. Это позволяет строить модели, которые с большей точностью отражают реальное состояние финансовых дел на предприятии. Моделировать различные ситуации в финансово-хозяйственной деятельности с целью определения путей развития организации при использовании конкретных ресурсов, сегментов рынка, знаний о конкурентной среде.

Вместе с тем следует учесть, что комплекс факторов не следует рассматривать как механическую сумму без учёта их взаимодействия, без выделения основных, определяющих, иначе выводы могут быть ошибочными.

В существующей практике научного исследования делается упор на нахождение влияния факторов на результирующий показатель. При этом не учитывают, что факторы, воздействуя на результирующий показатель, могут оказывать существенное влияние друг на друга. В этом случае возникает синергический или гасящий эффект. В частном случае возникает нейтральный, который не оказывает никакого влияния на исследуемый объект при определённом сочетании факторов.

Одной из форм противодействия внешним и внутренним угрозам становится подбор факторов таким образом, чтобы они оказывали гасящее воздействие друг на друга и это, в результате, позволяло избегать негативного воздействия на функционирование предприятия.

Надо отметить, что воздействия могут носить организующий и дезорганизующий характер на сферы финансово-хозяйственной деятельности коммерческой организации. При этом, усиливая или ослабляя финансовую устойчивость предприятия, которая, в первую очередь, связана спроцессом бесперебойного производства и реализации продукции, с финансовыми расчётами с поставщиками необходимых комплектующих, с потребителями продукции, товаров, услуг, с госорганами.

\subsection{3 Построение системы моделирования}

Для систематизации процесса воздействия различных факторов необходимо активно использовать процесс моделирования объектов. Причём моделирование объектов следует строить при выполнении следующих условий: наличия знаний об объекте исследования, определения сходства модели и оригинала, изучения отдельных сторон объекта проведение модельных экспериментов, на переносе знаний с модели на оригинал, на практической проверке полученных результатов.
Моделирование строится на изучении функционирования совокупности отдельных элементов системы, которые являются частью целого объекта исследования. В данном случае моделирование может выстраиваться на изучении отдельных сторон объекта или на рассмотрении целого объекта при условии, что для анализа привлекаем, с нашей точки зрения, наиболее важные элементы, а несущественные в конкретном случае отбрасываем.

Для получения в перспективе объективных данных необходима предварительная экспертная оценка отдельных элементов системы.

Актуальным фактом в исследовании объектов является определение уровня исследования. Так, эмпирический уровень исследует внешние проявления сущности объекта. В нашем случае в качестве таких проявлений выступают результирующие экономические показатели. Экономический подход как раз и характеризует данный уровень.

В любом случае научное исследование различных сторон объектов стремится выйти на теоретический уровень, который характеризует всесторонние грани объекта. Это позволяет определять причинно-следственные связи в изучаемом объекте, изыскивать средства для выхода предприятия из кризиса, и при успешном функционировании определять направления устойчивого развития организации.

\subsection{4 Выбор детерминированной модели}

В своём исследовании автор остановился на разработке детерминированной модели, определяющей функциональные связи между результирующим показателем и факторными признаками. Данная связь наиболее объективно в реальности характеризует процессы функционирования коммерческого предприятия и отражает эффективное использование ресурсов.

В качестве результирующего показателя взят доход организации. Факторными признаками являются показатели, характеризующие эффективное использование ресурсов.

$$
D=\sqrt{D \text { ф.л. } \times \Phi_{\text {отд. } \times R \text { ф.л. } \times} \Phi_{\text {вор. } \times \bar{R}}}
$$

где $D$ - доход предприятия от реализации продукции, работ, услуг; $\Phi_{\text {отд. }}-$ фондоотдача; $D_{\text {фл. }}-$ финансовые вложения на конец года; $R_{\text {ф.л. }}$ - рентабельность финансовых вложений; $Q_{\text {вор. }}-$ фондовооружённость; $\bar{R}-$ среднесписочное число работников.

Исходными данными для расчёта служат показатели, характеризующие эффективное использование ресурсов. Эти показатели представлены в таблице 1. 
Динамика эффективности финансовых ресурсов, p.

Table 1

The dynamics of the effectiveness of financial resources, ruble

\begin{tabular}{|l|c|c|c|}
\hline Показатели & \multicolumn{3}{|c|}{ год / уеаг } \\
\cline { 2 - 4 } Indicators & 2013 & 2014 & 2015 \\
\hline Доход от реализации продукции Revenue from product sales & 25346687 & 33645984 & 38420268 \\
\hline Финансовые вложения на конец года Financial investments at end of year & 3128620 & 2786578 & 1640242 \\
\hline Среднегодовая стоимость основных средств The average annual value of fixed assets & 1672041 & 1805479 & 1902242 \\
\hline Фондоотдача Сарital productivity & 15,159 & 18,635 & 20,197 \\
\hline Фондоемкость & 0,066 & 0,0537 & 0,0495 \\
\hline Фондовооруженность Саріtal intensity & 38001 & 40121 & 45291 \\
\hline Рентабельность финансовых вложений Return on investments & 8,102 & 12,074 & 23,424 \\
\hline $\begin{array}{l}\text { Среднесписочная численность работников, чел. } \\
\text { The аverage пumber of еmployees, реорle }\end{array}$ & 44 & \multirow{2}{*}{45} \\
\hline
\end{tabular}

Надо отметить, что основополагающими показателями, оказывающими существенное влияние на финансово-хозяйственную деятельность, являются основной, оборотный капитал и трудовые ресурсы. Причём трудом работников создаётся добавленная стоимость, поэтому при расширении системы учёта факторов, данные показатели необходимо учитывать в первую очередь, так как они вносят определяющий вклад в процесс производства. Влияние вышеперечисленных показателей на доход организации определяется методом цепной подстановки. Пример расчёта представлен в таблице 2. Надо отметить, что в ручном режиме расчёта возможна небольшая погрешность из-за не учёта знаков в показателях после запятой.

Таблица 2

Влияние на доход организации эффективных показателей

Table 2

The impact on income of the organization effective indicators

\begin{tabular}{|c|c|c|c|c|c|c|c|}
\hline Показатели / Indicators & \multicolumn{6}{|c|}{ Расчеты / Settlements } & 2014 year \\
\hline $\begin{array}{l}\text { Финансовые вложения на ко- } \\
\text { нец года / Financial invest- } \\
\text { ments at end of year }\end{array}$ & 3128620 & 2786578 & 2786578 & 2786578 & 2786578 & 2786578 & -1425053 \\
\hline $\begin{array}{l}\text { Фондоотдача } \\
\text { Capital productivity }\end{array}$ & 15,159 & 15,159 & 18,635 & 18,635 & 18,635 & 18,635 & 2601221 \\
\hline $\begin{array}{l}\text { Рентабельность финансовых } \\
\text { вложений } \\
\text { Return on investments }\end{array}$ & 8,102 & 8,102 & 8,102 & 12,074 & 12,074 & 12,074 & 5855124 \\
\hline Фондовооруженность & 38001 & 38001 & 38001 & 38001 & 40121 & 40121 & 890895 \\
\hline $\begin{array}{l}\text { Среднесписочное число } \\
\text { paботников / The average num- } \\
\text { ber of employees, people }\end{array}$ & 44 & 44 & 44 & 44 & 44 & 45 & 377110 \\
\hline $\begin{array}{l}\text { Доход организации } \\
\text { Revenue from product sales }\end{array}$ & 25346687 & 23921634 & 26522855 & 32377979 & 33268874 & 33645984 & 8299297 \\
\hline Показатели / Indicators & \multicolumn{6}{|c|}{ Расчеты / Settlements } & 2015 year \\
\hline $\begin{array}{l}\text { Финансовые вложения } \\
\text { на конец года / Financial in- } \\
\text { vestments at end of year }\end{array}$ & 2786578 & 1640242 & 1640242 & 1640242 & 1640242 & 1640242 & -7833109 \\
\hline $\begin{array}{l}\text { Фондоотдача } \\
\text { Capital productivity }\end{array}$ & 18,635 & 18,635 & 20,197 & 20,197 & 20,197 & 20,197 & 1060060 \\
\hline $\begin{array}{l}\text { Рентабельность финансовых } \\
\text { вложений / Return on investments }\end{array}$ & 12,074 & 12,074 & 12,074 & 23,424 & 23,424 & 23,424 & 10557085 \\
\hline Фондовооруженность & 40121 & 40121 & 40121 & 40121 & 45291 & 45291 & 2338565 \\
\hline $\begin{array}{l}\text { Среднесписочное число } \\
\text { paботников The average num- } \\
\text { ber of employees, people }\end{array}$ & 45 & 45 & 45 & 45 & 45 & 42 & -1348317 \\
\hline $\begin{array}{l}\text { Доход организации Revenue } \\
\text { from product sales }\end{array}$ & 33645984 & 25812875 & 26872935 & 37430020 & 39768585 & 38420268 & 4774284 \\
\hline
\end{tabular}

\section{Заключение}

Данная модель позволяет практически определять уровень влияния отдельного фактора на доход предприятия в стоимостном выражении, норму полезности отдельного ресурса, вид воздействий, выявлять организующие или дезорганизующие факты в работе организации. В целом вышеперечисленные экономические показатели являются индикаторами эффективного управления производством. 


\section{ЛИТЕРАТУРА}

1 Хорев А. И., Овчинникова Т. И., Кобелева С. В. Развитие конкурентоспособных инновационных производств на основе интенсификации // Экономика. Инновации. Управление качеством. 2014. № 2(7). С. 9-16.

2 Саликов Ю. А., Исаенко М. И. Особенности разработки процесса управления организационным развитием промышленного предприятия // Экономика. Инновации. Управление качеством. 2014. № 4 (9). С. 138

3 Пономарева Н. И. Развитие инновационной деятельности предприятий хлебопекарной промышленности // Актуальные проблемы гуманитарных и естественных наук. 2014. № 6-1. C. 287-291.

4 Кобелева С. В. Инновационная деятельность как источник повышения конкурентоспособности предприятий // Экономика. Инновации. Управление качеством. 2014. № 4 (9). С. 162-163.

5 Gorshkova L. A., Sovik L. E., Poplavskaya V. A. Methodology of organizational changes during the implementation of business-activity monitoring // International Journal of Business and Social Science. 2013. № 4. P. 104

6 Freeman A. “RENEWAL?”. Geopolitical Economy: After US hegemony, globalization and empire. Pluto Press, 2013. P. 153-186

7 Munro A., Bathelt H. "Innovation Linkages in New - and Old-economy Sectors in Cambridgeguelph-kitchener-waterloo (ontario)". Innovating in Urban Economies: Economic Transformation in Canadian City-regions. Ed. David A. Wolfe. Toronto: University of Toronto Press, 2014. P. 219-244

\section{СВЕДЕНИЯ ОБ АВТОРАХ}

Виктор М. Сидоров к. э. н., доцент, кафедра экономической безопасности и финансового мониторинга, Воронеж. гос. ун. инж. техн., пр-т Революции, 19, г. Воронеж, Россия, wictor.sidorov@yandex.ru

\section{КРИТЕРИЙ АВТОРСТВА}

Виктор М. Сидоров выполнил расчёты, обзор литературных источников по исследуемой проблеме, написал рукопись, корректировал её до подачи в редакцию и несёт ответственность за плагиат

\section{КОНФЛИКТ ИНТЕРЕСОВ}

Авторы заявляют об отсутствии конфликта интересов.

ПОСТУПИЛА 07.04.2016

ПРИНЯТА В ПЕЧАТЬ 05.05.2016

\section{REFERENCES}

1 Khorev A. I., Ovchinnikova T. I., Kobeleva S. V. The development of competitive industries on the basis of innovative labor intensification. Economy. Innovations. Quality Management. [Economy. Innovations. Quality Management] 2014, no. 2 (7), pp. 9-16. (in Russian).

2 Salikov Yu. A., Isaenko M. I. Features of the development process, management of organizational development of the industrial enterprise. Economy. Innovations. Quality Management. [Economy. Innovations. Quality Management] 2014, no. 4 (9), pp. 138 (in Russian).

3 Ponomareva N. I. Development of innovative activity of the enterprises of the baking industry. Actual problems of humanities and natural sciences. Aktual'nye problem gumanitarnykh i estestvennykh nauk [Russian Journal of Actual problems of humanities and natural sciences] 2014, no. 6-1, pp. 287291. (in Russian).

4 Kobeleva S. V. Innovation activity as a source of increasing the competitiveness of enterprises. Economy. Innovations. Quality Management. [Economy. Innovations. Quality Management] 2014, no. 4(9), pp. 162-163. (in Russian).

5 Gorshkova L. A., Sovik L. E., Poplavskaya V. A. Methodology of organizational changes during the implementation of business-activity monitoring. International Journal of Business and Social Science, 2013, no. 4, pp. 104

6 Freeman A. “RENEWAL?”. Geopolitical Economy: After US Hegemony, Globalization and Empire. Pluto Press, 2013, pp. 153-186

7 Munro A., Bathelt H. "Innovation Linkages in New - and Old-economy Sectors in Cambridge-guelphkitchener-waterloo (ontario)". Innovating in Urban Economies: Economic Transformation in Canadian City-regions. Ed. David A. Wolfe. Toronto, University of Toronto Press, 2014, pp. 219-244

\section{INFORMATION ABOUT AUTHORS}

Victor M Sidorov c. e. sc, associate Professor, department of economic security and financial monitoring, Voronezh state university of engineering technologies, Revolution Av., 19 Voronezh, Russia, wictor.sidorov@yandex.ru

\section{CONTRIBUTION}

Victor M Sidorov performed calculations, review of the literature on an investigated problem, wrote the manuscript, correct it before filing in editing and is responsible for plagiarism

\section{CONFLICT OF INTEREST}

The authors declare no conflict of interest.

RECEIVED 4.7.2016

ACCEPTED 5.5.2016 